\title{
S3 Vertebra
}

National Cancer Institute

\section{Source}

National Cancer Institute. S3 Vertebra. NCI Thesaurus. Code C33500.

The third of five sacral vertebrae. 